\title{
PROCEDIMENTI DI MISURAZIONE NELL'AMBITO DELLE DISCIPLINE ECONOMICO-SOCIALI
}

\author{
ANGELO ZANELLA $(*)^{1}$
}

RIASSUNTO. - 1) Si considera, in primo luogo, il procedimento di misura di una grandezza in ambito tecnologico nel quale è possibile accertare il valore vero della grandezza a meno di un errore aleatorio trascurabile rispetto a detto valore e quindi acquisire, ad un certo istante, in modo sostanzialmente esatto, lo stato di funzionamento di un processo.

Per le grandezze economiche, tipicamente di natura monetaria, si tratta di accertare, per un determinato periodo, l'ammontare totale o quello medio di una popolazione e si devono utilizzare, per la loro stima, il risultato di indagini campionarie.

L'errore medio di misura risulta quindi incrementato a causa della variabilità dovuta al campionamento. L'utilizzazione di schemi di campionamento efficienti assicura, però, che l'errore di misura di un totale o di una media aritmetica, rimane in pratica trascurabile rispetto al valore vero che può localizzarsi con sufficiente accuratezza. Viene illustrato questo aspetto con riferimento alla spesa famigliare annua media.

2) L'obiettivo diviene diverso se si è interessati non ad accertare una situazione in un determinato periodo di tempo, ma si vogliono delle previsioni per periodi futuri in base ad un modello economico e/o econometrico. In questo caso l'errore di misura e di campionamento può risultare incrementato in modo non direttamente valutabile a causa della non esatta conoscenza del modello interpretativo o anche per l'impossibilità di realizzare gli interventi correttivi sul sistema economico suggeriti dal modello. Un caso tipico viene ravvisato nella previsione della misura dell'inflazione.

3) Si osserva che la condizione di equilibrio di una economia di mercato può collegarsi alle scelte dei soggetti economici, cioè, in definitiva, alle scelte di individui che operano in accordo ad un'entità difficilmente definibile in termini generali e pertanto difficilmente "misurabile" indicata, come Utilità.

Sorge il quesito, suggerito ad esempio dalla teoria cinetica dei gas, che, come per un gas ha scarso interesse, ad esempio, la velocità di una singola molecola ma lo ha inve-

(*) Istituto Lombardo - Accademia di Scienze e Lettere, Università Cattolica del Sacro Cuore - Milano.

1 Ringrazio vivamente i colleghi Carlo Beretta, Laura Deldossi, Mario Faliva, Vittorio Benito Frosini, Giovanni Marseguerra, Piercarlo Nicola, Luigi Santamaria per il tempo dedicatomi e i pareri espressi durante la preparazione di questa relazione. 
ce il valore medio dell'energia cinetica delle molecole, espresso dalla temperatura, così può avere scarso interesse generale la misura dell'utilità percepita da un singolo consumatore, e si può invece pensare che abbia interesse qualche indicatore globale, che, argomento da approfondire-, si ritiene possa collegarsi ai prezzi di mercato.

4) Di fronte ad una pluralità di alternative soggette ad incertezza, che è una situazione tipica del "decisore" in ambito finanziario e d'impresa, la teoria di von Neumann-Morgenstern-Savage - che è stata soggetta a valutazioni critiche, revisioni e complementi - può essere utile per orientare verso le scelte che massimizzano l'utilità media, rispetto alle distribuzioni di probabilità ipotizzate, per le possibili conseguenze delle decisioni.

Nelle ricerche di mercato, poi, la formulazione della nozione di utilità diviene più concreta e le tecniche psicometriche spesso utilizzate, consentono di individuare il "prodotto" più utile.

Si è infine ricordato come è disponibile una metodologia statistico-probabilistica per la quantizzazione dei concetti, della quale si deve ancora approfondire l'impiego per la valutazione dell'utilità.

ABSTRACT. - 1) As starting point the measurement procedure of a given quantity - representing a typical problem in the technological area - is considered allowing one to ascertain the true value of the quantity except for a zero mean random error which in practice often may be assumed to have a unessential root mean squared error or repeatability standard deviation.

As for the quantities used in economic and social sciences - e. g..the economic quantities are often of monetary nature - with regard to a given finite population of elements, in general of human beings, a typical problem is of establishing the total amount of a chosen quantitative characteristic and /or the arithmetic mean, which have to be estimated on the basis of a sample survey. It follows that, in general, the random component of a single observation has a standard deviation greater than the one caused by repeatability owing to the presence of sampling variability. The use of efficient sample designs and a large sample size, however, ensure that the random errors of the estimates of the total amount and of the corresponding arithmetic mean may become unimportant with respect to their true values located through pertinent confidence intervals. An illustration is presented regarding the yearly mean expenditure of an Italian family.

2) The investigation purpose becomes more complex when we not only want to know the real situation in a given span of time but also when we want to forecast future trends according to an economic or an econometric model. In this case the global random error, due to measurement and sampling variability, may be affected by a systematic bias, which is not easy to be evaluated because of the lack of a complete and exact knowledge of the model. As an example the econometric models of inflation is quoted.

3) One can note that the equilibrium of a market economic system is connected to people's preferences and choices according to an abstract entity named Utility, which is difficult to be defined in general terms and thus to be measured.

Correspondingly, as in the gas kinetic theory the velocity of a single molecule can have no direct interest while on the contrary it does the mean value of the molecular kinetic energy expressed by temperature, likewise we can say that, in general, the Utility perceived by a single individual has little theoretical interest while a global Utility indicator of goods - which seems naturally linked to the market prices and deserves further 
investigation - could have a certain interest, e.g. for the aim of comparison

4) In the area of finance and enterprise management a decision maker is typically confronted with preference between decisions in the face of uncertainty. In this context the theory of von Neumann - Morgenstern - Savage - which has been the subject of some criticism, revision and completion - may at times appear to be useful. More precisely, assuming a set of possible monetary consequences and some alternative probability distributions on it, the theory directs a decision maker towards the choice which maximizes the expected value or mean Utility within the values corresponding to the alternative probability distributions .

In marketing research the notion of Utility assumes a more concrete character and the psychometric techniques, which are often used, allow one to point out the more useful product..

Finally the available statistical - probabilistic theory is mentioned which permits the quantization of abstract notions or concepts and whose appropriateness and use, with respect to economic utility evaluation, have still to be examined attentively.

\section{PREMESSA: ANALOgIE CON I PROCESSI INDUSTRIALI E DATI STATISTICI DISPONIBILI}

Questa relazione conclusiva vuole sottolineare la prospettiva applicativa presente nella giornata di studio. Essa consiste nell'avere la consapevolezza che la teoria economica, che fa da sfondo all'Economia matematica e all'Econometria, quando è integrata dai riscontri sperimentali concernenti un determinato sistema economico - il riferimento implicito sarà nel seguito costituito da una "economia di mercato", diciamo in senso lato, quale è quella Italiana -, consente di descrivere e comprenderne gli aspetti essenziali presenti, di delinearne i possibili sviluppi futuri e di suggerire eventuali interventi correttivi per raggiungere un particolare scopo. Sussiste un'analogia con quanto accade nella conduzione e nel controllo di un sistema produttivo.

Quest'ultimo è in effetti conforme ad un progetto tecnico, che discipline sostanziali, quali, ad esempio, la fisica e la chimica, hanno indicato come adatto all'ottenimento di specifici risultati, mentre il sistema economico è sostanzialmente subito. Tuttavia anche la conduzione e il controllo di un processo tecnologico richiede di accertare con continuità lo stato di buon funzionamento dello stesso mediante l'osservazione di numerose variabili e, se necessario, di eseguire degli interventi correttivi, ciò che accade quando si è riscontrato uno stato del sistema che si prevede possa compromettere il raggiungimento degli obiettivi. 


\subsection{I dati dell'Istituto Centrale di Statistica}

Lo studio sperimentale per l'Italia dei principali aspetti sociali ed economici è svolto, in parte, direttamente dall'ISTITUTO CENTRALE DI STATISTICA (ISTAT) e, comunque, è reso possibile dalla raccolta e pubblicazione sistematiche di dati assicurata dall'ISTAT. Detto Istituto pubblica ogni anno un grosso volume che raccoglie i dati di un anno, l'Annuario dell'ISTAT; molto rilevante è, poi, il Bollettino mensile di Statistica nel quale sono mensilmente presentati i dati attinenti ai seguenti temi:

RISORSE ED ECONOMIA: Agricoltura e Foreste, Industrie (Numeri indici della produzione industriale, Numeri indici del fatturato e degli ordinativi, Produzioni industriali), Trasporti, Commercio interno, Commercio con l'estero, Credito, Mercato monetario finanziario, Prezzi, Lavoro, Finanze pubbliche.

\subsection{I dati della Banca d'Italia}

Per l'analisi economica i dati dell'ISTAT sono completati da quelli forniti dalla Banca d'Italia e contenuti nelle principali pubblicazioni economiche della stessa costituite dalla Relazione Annuale, il Bollettino Statistico e il Quadro di Sintesi, con cadenza trimestrale, integrati dai Supplementi al Bollettino Statistico, con periodicità prevalentemente mensile. Per finalità di analisi economica la Banca d'Italia effettua indagini campionarie periodiche quali "Indagine sui bilanci delle famiglie Italiane", con cadenza biennale, "Indagine sulle imprese industriali e dei servizi", con cadenza annuale,"Sondaggio congiunturale sulle imprese industriali e dei servizi", con cadenza annuale, "Indagine sulle aspettative di inflazione e crescita", con cadenza trimestrale.

\section{L'ERRORE DI MISURA}

\subsection{Misure di tipo fisico}

Come riferimento si assumerà il procedimento di misurazione tipico in ambito tecnologico, che indichiamo, per semplicità, di tipo fisico. Esso comporta: 1) la scelta di un'entità o unità di tipo fisico; 2) la scelta di un "aspetto" della stessa che si vuole misurare e deve coinci- 
dere con la determinazione di una grandezza (tipiche e ben note sono, ad esempio, classi di grandezze come quella delle masse, delle lunghezze, delle durate di tempo, ecc.);3) la scelta di un'altra entità fisica, con presente la stessa grandezza, la cui determinazione è assunta quale unità di misura; 4) la definizione di un procedimento che consente di stabilire quante volte l'unità di misura è contenuta nella grandezza da misurare, numero che ne esprime la misura rispetto ad una prescelta unità.

$\mathrm{Si}$ ammette, pertanto, che la misura di una grandezza fisica sia un incognito valore $\eta$, oggetto della misura, e che, almeno concettualmente, sia possibile ripetere, quindi attuare esattamente nelle stesse condizioni, la misurazione quante volte si vuole. Si può in corrispondenza definire un processo materiale di misurazione che porta ad osservazioni della misura della grandezza - assunta per semplicità quantitativa continua, diciamo $Y$ - che soddisfano la seguente relazione:

$y_{j}=\eta+e_{j}$

$j=1,2, \ldots, m$, dove $e_{j}$ sono errori casuali, ritenuti determinazioni di variabili casuali $E_{j}$ stocasticamente indipendenti, con la stessa distribuzione di probabilitâ, valore atteso $M\left(E_{j}\right)=0, j=1,2, \ldots, m$, e $\operatorname{Var}(Y)=\sigma_{r}^{2}$.

Secondo il precedente modello interpretativo le misure ripetute di una stessa grandezza non sono eguali, in virtù degli errori aleatori. La variabilità media delle misure è riassunta dalla loro varianza $\sigma_{r}^{2}$, chiamata varianza di Ripetibilità. Si giunge alla nozione di Riproducibilità di un procedimento di misurazione se si tiene conto delle possibili altre fonti di variabilità accidentale, quali i diversi operatori, i diversi laboratori, ecc. . In corrispondenza una semplice generalizzazione della (1) diviene:

$y_{i j}=\eta+b_{i}+e_{i j}=\eta+\beta+\left(b_{i}-\beta\right)+e_{i j}=\eta+\beta+d_{i j}$

avendo aggiunto e tolto il valore medio costante $\beta$ e posto $d_{i j}=\left(b_{i j}-\beta\right)+$ $e_{i j}$, inoltre si suppone che la misura di ogni unità sia, ad esempio, ripetuta da $i=1,2, \ldots, I$, operatori, eventualmente in altri laboratori, ecc., $e_{i j}$ sono $n=I \times m$ errori accidentali, esattamente del tipo visto per il modello (1), descritti da variabili fra loro stocasticamente indipendenti, con la stessa distribuzione di probabilità, con media nulla e varianza $\sigma_{r}^{2} ;\left(b_{i}-\beta\right)$ sono degli errori aleatori descritti dalle variabili casuali $\left(B_{i}-\beta\right),-$ per un determinato $i, B_{i}$ idealizza una scelta casuale da un "universo" di infinite possibili condizioni di sperimentazione - stocasticamente indipendenti fra 
loro e dalle precedenti, con distribuzione di probabilità di media nulla e comune varianza $\sigma_{B}{ }^{2}$. Si definisce varianza di Riproducibilità la quantità:

$\sigma_{R}^{2}=\sigma_{B}^{2}=\operatorname{Var}\left(B_{i}\right)$

In conclusione il procedimento di misurazione comporta, in accordo alla (2), per un'unità sperimentale di osservare in luogo del valore vero $\eta$ un valore $\eta^{*}=\eta+\beta+d_{i j}$, caratterizzato dalla eventuale "distorsione lineare" media $\beta$, oltre che da un errore aleatorio totale $d_{i j}$, la cui varianza risulta pari alla somma della varianza di ripetibilità e di quella di riproducibilità, cioè:

$\sigma_{T}^{2}=\sigma_{r}^{2}+\sigma_{R}^{2}$

da cui lo scarto quadratico medio

$\sigma_{T}=\left(\sigma_{r}^{2}+\sigma_{R}^{2}\right)^{1 / 2}$.

Lo schema (2) consente, di ottenere delle stime corrette di $\eta^{*}$, non di $\beta$, parametro "confuso" con $\eta$, di $\sigma_{r}^{2}$ e di ${\sigma_{R}}^{2}$, quindi di $\sigma_{T}^{2}$.

La precedente misura della variabilità accidentale totale è utilizzata nel controllo di una caratteristica quantitativa, di un determinato sistema produttivo statisticamente stabile, - ipotizzato un valore per la media - per chiarire quale è, a meno degli errori di misura, la situazione vera e trarne le conclusioni, e anche, assumendo il sistema invariante, per prevedere, osservato un valore $y_{t-1}$ al tempo $(t-1)$, quale potrebbe essere $y_{t}$ al successivo istante di osservazione in conseguenza degli errori di misura. Si tratta di valutazioni ritenute convenzionalmente certe, che possono, però, formularsi anche nel senso che hanno una probabilità prossima ad $1 \mathrm{di}$ essere valide.

\subsection{L'errore nelle indagini campionarie in ambito economico:}

campionamento casuale semplice

I dati di carattere economico offerti dall'ISTAT sono tipicamente basati, ovvero integrati, mediante indagini campionarie. E'questo l'aspetto tipico che caratterizza il contesto in cui ora ci si pone e lo distingue da quello tecnologico, e precisamente: $a$ ) le unità sperimentali (per- 
sone, famiglie, imprese, ecc.) oggetto di studio costituiscono un insieme, diciamo, di $N$ elementi già esistenti, la cosiddetta popolazione $P ; b$ ) se, ad esempio, si considera un carattere quantitativo $Y$, interessa misurare il totale presente nella popolazione o la corrispondente media aritmetica; c) tranne che nel caso di censimento, la corrispondente osservazione completa non è possibile per motivi di costi e di tempo e si deve ricorrere ad un sottoinsieme della popolazione, il cosiddetto campione, adatto a rappresentarla su scala ridotta, cioè mediante l'osservazione di $n<N$ unità della popolazione $\mathrm{e}$, in generale, estratto casualmente da $P$. Un tipico campionamento è quello cosiddetto casuale semplice senza reinserimento (CCS) che richiede un procedimento operativo di base, presente anche nei disegni di campionamento più complessi, che comporta l'estrazione di $n$ diverse unità sperimentali, con la stessa probabilità di estrazione qualsiasi sia l'n-pla, e verrà esaminato con qualche particolare per chiarire in modo semplificato le peculiarità delle misure ottenute su base campionaria.

Si indichi con $\eta_{b}, b=1,2, \ldots, N$, il valore vero di un carattere quantitativo proprio della generica unità sperimentale di $P$ e siano :

$$
\bar{\eta}=\left(\Sigma_{b=1}^{N} \eta_{b}\right) / N ; \sigma_{\eta}^{2}=\Sigma_{b=1}^{N}\left(\eta_{b}-\bar{\eta}\right)^{2} / N
$$

rispettivamente la media aritmetica e la varianza di tali valori. Si supponga di utilizzare un CCS di $n=m \times I$ elementi dalla popolazione $P$, con $n / N$ trascurabile, per semplicità, ai quali, ad esempio corrisponda l'attuazione di $n$ interviste ripartite fra $I$ operatori, ciascuno esecutore di $m$ interviste concernenti $m$ unità sperimentali diverse. La misura $\eta_{i j}$ di $Y$ eseguita sulla generica unità estratta può essere descritta dal modello:

$y_{i j}=\eta_{i j}+b_{i}+e_{i j}=\eta_{i j}+\beta+\left(b_{i}-\beta\right)+e_{i j}=\eta_{i j}+\beta+d_{i j}$

$i=1,2, \ldots, I, j=1,2, \ldots, m$, dove per ogni coppia di valori $(i, j)$ che identifica uno degli $n$ elementi del campione, $\eta_{i j}$ è la determinazione di una variabile casuale marginale $H$ di media $\bar{\eta}$, e varianza $\sigma_{\eta}^{2} ; b_{i}$ esprime l'errore dell'operatore $i$-mo che si assume sia estratto a caso da un "universo", diciamo per semplicità, di infiniti operatori, ed i valori $\left(b_{i}-\beta\right)$, al variare di $i$ si ritengono determinazioni stocasticamente indipendenti, fra loro e rispetto ad $H$, di variabili casuali $\left(B_{i}-\beta\right)$ ciascuna di media 
nulla e varianza $\sigma_{B}^{2} ; e_{i j}$ rappresenta l'errore di misura per l'unità sperimentale $j$-ma esaminata col contributo dell'operatore $i$-mo, e si assume che i valori $e_{i j}$ siano determinazioni di variabili casuali, stocasticamente indipendenti fra loro e rispetto ad $H$ e $\left(B_{i}-\beta\right)$, di media nulla e varianza $\sigma_{E}^{2}$.

Se si tiene conto del campionamento, in base al modello (5), la grandezza $Y$ in studio, anche a prescindere dagli errori $b_{i}, e_{i j}$, non presenta un unico valore "vero" $\eta$, bensì il valore $\eta_{i j}, i \in\{1,2, \ldots, I\}$, $j \in\{1,2, \ldots, m\}$ corrispondente all'elemento individuato dalla coppia di valori fra gli n elementi estratti col campione.

Si osserva, dunque, la quantità :

$\eta_{i j}^{*}=\eta_{i j}+\left(\beta+d_{i j}\right)$

caratterizzata da un'eventuale distorsione lineare $\beta$ - errore sistematico oltre che da una deviazione accidentale $d_{i j}$, che modificano il valore $\eta_{i j}$ proprio dell'unità $(i, j)$ del campione CCS che, appunto come conseguenza dell'estrazione campionaria, ha varianza $\sigma_{\eta}^{2}$. In definitiva, in virtù delle assunzioni fatte, consegue:

$\operatorname{Var}\left(Y_{i j}\right)=\sigma_{T}^{2}=\sigma_{\eta}^{2}+\sigma_{B}^{2}+\sigma_{E}^{2}$

da cui lo scarto quadratico medio $\sigma_{T}=\left(\sigma_{\eta}^{2}+\sigma_{B}^{2}+\sigma_{E}^{2}\right)^{1 / 2}$.

Il confronto fra le espressioni $(\overline{3})$ relativa alle misure fisiche e la (6), relativa alla misura nell'ambito di un campionamento da $N$ unità già disponibili, mette in luce una diversità essenziale. In questo secondo caso, descritto dalla (6), la variabilità accidentale complessiva, da associare alla misurazione di una grandezza, deve anche includere la componente di variabilità strutturale $\sigma_{\eta}^{2}$, propria della popolazione $P$, si veda sul tema l'articolo di P.P. Biemer, D. Trewin (1997).

La relazione (6) serve a ottenere una valutazione di insieme delle fonti di variabilità accidentale che hanno, in primo luogo, un effetto sulla stima del vero valore di interesse - tipicamente un totale o una media aritmetica - attinente all'intera popolazione. Infatti, come si è visto, $\sigma_{\eta}^{2}$ tiene conto della variabilità di campionamento, $\sigma_{B}^{2}$ della variabilità collegata agli operatori che svolgono l'indagine, $\sigma_{E}^{2}$ degli errori di misurazione, costituiti dalla differenza fra il vero valore della caratteristica da misurare su una data unità statistica ed il valore direttamente osservato o registrato: sono possibili differenze dovute allo 
stesso rispondente di un questionario, ma anche dovute agli errori di registrazione su supporto magnetico o di codifica dei quesiti. Esistono, però, altre possibilità di errore che non sono incluse nella relazione (5) e si cerca, comunque, di non trascurare nelle indagini reali fra i quali gli errori di "copertura" concernenti la definizione della popolazione, gli errori da mancate risposte, ecc., che possono contribuire alla componente sistematica.

Un'analisi di dettaglio mostra precisamente il carattere peculiare, diciamo "personalizzato", delle rilevazioni in ambito economico e sociale, nel senso che esse sono tipicamente collegate all'adeguamento di un soggetto alle richieste di un questionario, ciò che consente di ridurre, con riferimento al modello (5), la distorsione in media $\beta$ e la componente di variabilità accidentale riassunta nella $(6) \mathrm{da}\left({\sigma_{B}}^{2}+\sigma_{E}{ }^{2}\right)$. In corrispondenza nelle più importanti indagini campionarie condotte dall'ISTAT, come quella attinente ai consumi famigliari medi, si provvede ad un'accurata fase preliminare di revisione e controllo dei dati che consente di ignorare la distorsione in media e di considerare dominante nella (6) la variabilità dovuta al campionamento, espressa da $\sigma_{\eta}^{2}$.

In conclusione la varianza delle stime ottenute per i totali o per le medie viene alla fine collegata alla sola variabilità di campionamento. Nel seguito si riterrà accettabile questa semplificazione.

\subsection{Disegni di Campionamento più complessi}

Si è iniziato con l'esame di uno schema di campionamento particolarmente semplice (CCS ) per avvicinare, senza dettagli concettualmente superflui, il problema della variabilità accidentale, che caratterizza i dati utilizzati nell'ambito delle discipline economico-sociali. In effetti, in indagini reali, del tipo, ad esempio di quelle condotte dall'ISTAT, proprio la presenza nei dati di "una componente di variabilità accidentale dovuta al campionamento" richiede di ricorrere a strategie campionarie complesse. Ciò proprio per contrastarne l'effetto sulla stima della quantità complessiva, totale o media aritmetica, della popolazione $P$ che si deve approssimare mediante l'utilizzazione di un sottoinsieme di $n$ dati campionari. Si pone, quindi, l'esigenza, di utilizzare delle tecniche di campionamento che consentano di ottenere delle stime almeno tendenzialmente corrette e sufficientemente efficienti, vale a dire con una "piccola varianza".

$\mathrm{Si}$ aggiunge al riguardo qualche cenno. 
Campionamento stratificato. Esso comporta, prima di procedere all'estrazione del campione, di suddividere la popolazione in due o più gruppi secondo una o più caratteristiche note delle unità statistiche (ad esempio caratteristiche geografiche quali: regioni, provincie, comuni ovvero demografiche quali: ampiezza della popolazione, composizione per età della stessa, ecc.).

Campionamento a due o più stadi. Si ricorre a questo tipo di campionamento quando non sia disponibile una lista complessiva delle unità della popolazione. Un esempio di tale situazione è dato dall'anagrafe per la quale non esiste un unico archivio nazionale in quanto essa è suddivisa negli 8103 comuni italiani.

In un caso come questo si procede dapprima ad estrarre un campione (CCS) di comuni, unità di primo stadio, e quindi, per ogni comune selezionato, si estrae, ad esempio, un campione casuale (CCS) di famiglie da ciascuna lista anagrafica di comune, unità di secondo stadio.

Selezione delle unità con probabilità differenti. La probabilità di estrazione di un'unità non è la stessa per tutte le unità della popolazione. Si ricorre a questa tecnica quando sussiste il motivo di ritenere che alcune unità statistiche apportino maggiori informazioni rispetto ad altre e si vuole aumentare la probabilità che siano selezionate ( ad esempio se si conosce il numero di addetti per una lista di imprese delle quali si vuole selezionare un campione su cui rilevare il fatturato, è ragionevole selezionare le imprese con probabilità proporzionale al numero di addetti di ciascuna, qualora si ritenga che il fatturato delle imprese più grandi sia maggiore di quello delle più piccole).

\subsection{Il software per lo studio dello scarto quadratico medio dell'errore nei disegni campionari complessi}

Si tratta di strumenti informatici essenziali nell'ambito di indagini campionarie di respiro nazionale come quelle condotte dall'ISTAT e dalla Banca d'Italia. Al riguardo si ricorda la sintesi articolata e interessante, per il periodo in cui è stata scritta, di J. Blum (1983).

L'ISTAT ha messo a punto e sta perfezionando una propria procedura informatica, cosiddetta GENESEES.

Si veda ISTAT: "Produzione delle stime di interesse e valutazione degli errori campionari” all'indirizzo web http://www.istat.it/stru- 
menti/metodi/software/produzione_stime e per maggiori particolari tecnici: M. Scannapieco, D. Zardetto e G. Barcaroli (2007).

Nella prima fonte ISTAT sopraccitata si fa presente che "Le indagini ISTAT vengono realizzate attraverso disegni di campionamento complessi spesso basati su più stadi di selezione, sulla stratificazione delle unità, e sulla possibile selezione delle stesse con probabilità variabili di inclusione e senza reinserimento. Inoltre le stime vengono determinate mediante l'utilizzo di stimatori con ponderazione vincolata.

Le metodologie standard adottate dall'ISTAT per la valutazione degli errori campionari delle stime uscenti dalle varie indagini campionarie si basano: 1) Sulla determinazione dell'espressione analitica dello stimatore della varianza in relazione al campionamento adottato; 2) Sul metodo di linearizzazione di Woodruff nel caso in cui gli stimatori adottati sono funzioni non lineari dei dati campionari. Sulla base di dette metodologie l'ISTAT ha sviluppato il software generalizzato GENESEES, che viene correntemente utilizzato per le stime degli errori campionari delle stime prodotte nelle diverse indagini sulle famiglie e sulle imprese".

Nella seconda fonte ISTAT richiamata si mostra, in termini astratti ma precisi, il punto focale del software: esso si fonda sullo stimatore non distorto lineare di Horvitz-Thompson, definito, al riguardo del totale della caratteristica di interesse $Y$ dall'espressione:

$y_{H T}=\Sigma_{K \in S} \quad \delta_{k} y_{k}$

dove $S$ indica l'insieme di $n$ valori ordinatamente associati alle $n$ etichette che identificano le unità estratte, $\delta_{k}=1 / \pi_{k}$, con $\pi_{k}>0$, la probabilità che l'unità k-ma sia inclusa nel campione.

L'accuratezza delle stime ottenute con il metodo di HorvitzThompson può essere migliorata mediante l'utilizzo di stimatori alternativi. Gli stimatori cosiddetti di calibrazione o di riponderazione, considerati nel software dell'ISTAT un oggetto, sono solo asintoticamente corretti. Si mostra che tuttavia assicurano un "guadagno" di efficienza. Essi si basano su un sistema di pesi calcolato in modo piuttosto complesso. I pesi finali $w_{k}$ si ottengono risolvendo un sistema di minimo vincolato, in cui la funzione da minimizzare è una funzione di distanza tra i pesi diretti $\delta_{k}$ ed i pesi finali $w_{k}$ ed i vincoli sono rappresentati da condizioni di eguaglianza delle stime campionarie di variabili ausiliarie 
con i rispettivi totali noti della popolazione, che sono definiti da fonti esterne all'indagine. Lo stimatore di calibrazione è formalmente del tipo indicato dalla relazione (7), nella quale i pesi diretti $\delta_{k}$ sono sostituiti da quelli finali $w_{k}$.

Conoscendo la dimensione $N$ della popolazione è poi possibile passare in modo piuttosto agevole dagli stimatori del totale e della relativa varianza a quelli della media aritmetica e relativa varianza.

\subsection{Definizione di un intervallo che contiene l'incognita misura vera per un determinato periodo di tempo con una assegnata probabilità}

La teoria probabilistica del campionamento consente di stabilire un intervallo tale che, in base ai risultati campionari, sussista una probabilità da ritenere prossima ad un valore, diciamo $p$, che esso contenga la incognita media aritmetica vera $\bar{\eta}$, se questa è la grandezza di interesse, ovvero $\tau=N \bar{\eta}$ se lo studio concerne il totale.

Se, ad esempio, si assume che gli stimatori della misura di interesse - media aritmetica o totale $\tau$ - seguano una distribuzione normale un tale intervallo, per $p=0,95$, cioè, con una probabilità prossima al $95 \%$ di contenere l'incognita misura, risulta del tipo:

$\left\{\hat{Y}\left(y_{k}, k=1,2, \ldots n\right)-1,96 \hat{\sigma}(\hat{Y}), \quad \hat{Y}\left(y_{k}, k=1,2, \ldots n\right)+1,96 \hat{\sigma}(\hat{Y})\right\}$

dove $\hat{Y}\left(y_{k}, k=1,2, \ldots n\right)$ è lo stimatore della grandezza di interesse, $\hat{\sigma}(\hat{Y})=\sqrt{\widehat{\operatorname{var}}(\hat{Y})}$

è la stima dello scarto quadratico medio di $\hat{Y}$, essendo $\widehat{\operatorname{var}}(\hat{Y})$ la stima della corrispondente varianza.

Si può dare maggiore concretezza a quanto è stato sopra accennato considerando un caso reale. Esaminiamo brevemente a tale fine la valutazione della spesa media mensile delle famiglie italiane attuata ogni trimestre e, in conclusione per l'intero anno, dall'ISTAT mediante un'indagine campionaria. Si tratta di un indicatore importante dato che ad esso fa riferimento il calcolo del prodotto interno lordo ed in tempi recenti ha acquisito particolare rilevanza per stabilire la soglia conven- 
zionale di povertà, che nel 2006 per una famiglia di due componenti è stata fissata in $€ 970,34$. Per famiglie di ampiezza diversa il valore convenzionale si ottiene dal precedente applicando un'opportuna scala di equivalenza. In conclusione per il 2006, secondo la stima dell'ISTAT che, come già accennato, pone in atto controlli rivolti a ridurre gli errori non direttamente dovuti al campionamento, le famiglie Italiane in condizioni di povertà relativa rappresentano l'11,1\% delle famiglie residenti. Per maggiori approfondimenti sul tema della misura della povertà in Italia, si può vedere M. Marigliani (2004).

L'indagine sulla spesa media famigliare condotta dall'ISTAT si basa su un campione a due stadi: unità di primo stadio sono i comuni, quelle di secondo stadio sono le famiglie. L'indagine coinvolge complessivamente 467 comuni. Il territorio italiano è stato suddiviso in 227 strati, 107 costituiti da singoli comuni, tipicamente capoluoghi di provincia, con l'eccezione di 4, cosiddetti autorappresentativi (AR), 120 costituiti, invece, raggruppando, all'interno del medesimo strato, comuni della stessa regione aventi la stessa tipologia demografica ed in modo che ogni strato abbia la stessa ampiezza demografica finale (comuni non autorappresentativi: NAR). Le unità di secondo stadio sono le famiglie: il disegno di campionamento prevede che ne siano intervistate intorno a 28000 l'anno, ovvero circa 2358 al mese, residenti nei 227 comuni che di volta in volta partecipano all'indagine con carattere continuativo nel corso dell'anno. Sotto si riportano i valori medi annuali nazionali per il 2004, 2005, 2006, in € pubblicati dall'ISTAT, l'ampiezza dei corrispondenti intervalli \pm [ ] cosiddetti di confidenza - al livello di probabilità indicativo del $95 \%$ - in base alla precedente relazione (8), il valore percentuale della loro ampiezza rispetto al valore medio del consumo famigliare annuale nazionale.

Tabella1. Stima campionaria nazionale della spesa media famigliare mensile [intervalli di confidenza assoluti $(€$ e percentuali rispetto a tale stima], $p \cong 0.95$

\begin{tabular}{cccc}
\hline & 2004 & 2005 & 2006 \\
\hline$\hat{y}$ & $2.381[57: 2.4 \%]$ & $2.398[51: 2.1 \%]$ & $2.461[61: 2.5 \%]$ \\
\hline
\end{tabular}

In conclusione si trova, dunque, che l'incognito valore della misura della media è individuato, con probabilità del $95 \%$ circa, a meno di un errore poco più dell' $2 \%$ rispetto all'ordine di grandezza fornito nel- 
l'anno dalla media generale nazionale. Si tratta di una "buona approssimazione" del valore vero, in genere ritenuta appropriata anche in ambito tecnologico, per valutare ad un certo tempo la situazione di interesse.

\subsection{La previsione dei valori futuri}

Rispetto al problema di misurare il valore di una particolare grandezza, in un determinato periodo di tempo (ad esempio la media aritmetica o il totale di una popolazione) completamente diverso è quello di prevedere il valore in un periodo futuro. Tipico della previsione mediante metodi statistici è l'utilizzazione di un modello dinamico, cioè dipendente dal tempo, nel quale i valori passati sono collegati con i valori futuri.

Si consideri, anche in questo caso, un esempio molto semplice, per percepire gli aspetti essenziali senza complicazioni ridondanti, e $t$ indichi, in unità convenzionali, il tempo alla fine di un periodo considerato $t=1,2 \ldots, T$. Con riferimento, per fissare le idee, alla spesa media del consumo famigliare annuale si consideri il modello:

$y_{t}=\hat{\eta}_{0}+\alpha t+\varepsilon_{t}$

dove $y_{t}$ sono i valori campionari, $\eta_{0}, \alpha$ sono incognite costanti, con stime $\hat{\eta}, \hat{\alpha}, \hat{\alpha}$ ottenute, ad esempio, con il principio dei minimi quadrati da tali valori, per $t=1,2 \ldots, T-1, \varepsilon_{t}$ sono errori globali supposti determinazioni di variabili casuali di media nulla e varianza $\sigma_{s}^{2}$ e fra loro stocasticamente indipendenti. Al tempo (T-1) la relazione (9) consente la previsione al tempo $T, T>1$, come :

$\hat{y}_{T}^{p}=\hat{\eta}_{0}+\hat{\alpha} T$

che coincide con la stima della media del secondo membro della (9) condizionata per $t=T-1$. Infatti, la (10) si ottiene: 1$)$ prendendo come predittore di $y_{t}$ in $t=T$ la media dell'espressione (9) condizionata per $t=T-1$, tenuto conto che l'assunzione, di errori indipendenti di media nulla, comporta di ritenere nulla, condizionatamente al valore $\varepsilon_{T-1}$, la media dell'errore di previsione $\varepsilon_{T}$, dato dalla differenza tra il valore vero $y_{T}$ e quello ottenuto dal modello, privato dell'errore; 2 ) utilizzando per i parametri le stime disponibili, in base ai dati ottenuti fino a $t=$ T-1, nel calcolo della media condizionata. 
Risulta, pertanto:

$\left(y_{T}-\hat{y}_{T}^{p}\right)=\left(\eta_{0}-\hat{\eta}_{0}\right)+(\alpha-\hat{\alpha}) T+\varepsilon_{T}$

con variabilità media espressa dall'errore quadratico medio dato da $M\left[\left(y_{T}-\hat{y}_{T}^{p}\right)^{2}\right]$, che dipende dalla variabilità media delle stime $\hat{\eta}_{0}, \hat{\alpha}$, oltre che da quella dell'errore imprevedibile $\varepsilon_{T}$. Questo se il modello (9) è esatto; se invece, ad esempio, non si è tenuto conto del contributo $\gamma Z_{t}$, con $\gamma$ un'incognita costante, di una variabile esplicativa - nell'esempio considerato potrebbe essere il tasso di disoccupazione - qualora $Z_{t}$ siano delle variabili casuali di media nulla, varianza $\sigma_{Z}^{2}$ stocasticamente indipendenti dagli errori $\varepsilon_{t}$, il precedente errore quadratico medio risulterebbe incrementato della quantità $\gamma^{2} \sigma_{Z}^{2}$.

In conclusione l'errore di previsione può risultare ben maggiore dell'errore campionario e di misura per il fatto che non sempre si conosce esattamente, in base alla teoria economica, un modello interpretativo appropriato completo e stabile della grandezza di interesse, sia per quanto attiene alle variabili esplicative che alla forma matematica.

Ma quanto è più rilevante è che la previsione in termini probabilistici mediante un intervallo di confidenza risulta praticamente impossibile poiché rimane in definitiva incognito l'errore dovuto alla non completa conoscenza del modello interpretativo.

Questo si presenta per casi importanti fra i quali si può porre il modello econometrico dell'inflazione. A riguardo ricordiamo, in primo luogo, il modello collegato al Servizio Studi della Banca D'Italia, si veda: V. Roffia ed A. Zaghini (2008), nel quale si discute ed esamina un modello di inflazione che si fonda principalmente su variabili esplicative di carattere finanziario. Si afferma in conclusione che "L'evidenza empirica mostra come gli episodi di forte crescita monetaria associati a sostenuti incrementi degli indici azionari e dei prezzi delle case ed una elevata dinamica del credito al settore privato, tendono ad essere seguiti da significativi incrementi dell'inflazione. Da un punto di vista di policy questi risultati implicano che nel breve periodo le dinamiche degli aggregati monetari devono essere monitorate congiuntamente ad altri indicatori."

L'altro modello che si segnala è quello elaborato dal Centro Studi della Confindustria, si veda C. Rapacciuolo (2003). L'approccio tende ad essere auto-esplicativo: il punto di partenza del modello econome- 
trico è la decomposizione dell'indice generale dei prezzi al consumo in una componente essenziale, la cosiddetta core inflation che tiene conto di tendenze di più lungo termine, e almeno due componenti più "volatili", ad esempio, rappresentate dai prezzi al consumo degli alimenti freschi e da quelli dei prodotti energetici.

\section{Misura DELL'UTILITÀ}

Si tratta di un argomento complesso che per un esame particolareggiato avrebbe richiesto una parte a sé stante non trascurabile del convegno. Se ne può quindi solo accennare.

\subsection{L'utilità come carattere ordinale}

La nozione o concetto di utilità si riferisce alla struttura psicologica di un soggetto in grado di stabilire delle preferenze nella scelta tra possibili alternative o conseguenze oggettive.

Si considera precisamente un insieme $X$ di elementi (candidati in una elezione, paniere di beni di consumo, offerte di lavoro, proposte di investimento, ecc.) per ciascuna coppia dei quali un soggetto può fare un confronto di preferenza (anche di indifferenza) che consiste nello stabilire per l'attributo Utilità quale dei due elementi è preferibile all'altro o se entrambi devono considerarsi eguali - assegnazione della modalità "maggiormente preferito" ad uno se non vale l'eguaglianza di entrambi. Si suppone inoltre che il confronto di preferenza assicuri una scala ordinale cosicchè se $z, y$ sono elementi di $X$ e $z$ è preferito ad $y$, ciò che si suole indicare come $z-y$, esiste una funzione reale $u(x), x \in X$ per cui vale:

$u(z)>u(y)$

per ogni coppia di $z$ - $y$ dove $u(x)$ è definita a meno di una funzione $f[\cdot]$ strettamente monotona, si veda ad esempio D. H. Krantz, R. Luce, P. Suppes, A.Tversky (1971).

In ambito economico è tipico associare alle possibili alternative/conseguenze oggettive dei valori monetari che di per sé nulla dicono sulla corrispondente utilità soggettivamente percepita da chi decide la scelta. 


\subsection{La misurazione dell'utilità media}

"Per ogni sistema di prezzi ciascun consumatore sceglie un paniere di consumo che massimizza la propria funzione di utilità, subordinatamente al vincolo di bilancio, e cioè alla condizione per la quale il costo del paniere scelto non deve eccedere il valore delle dotazioni ai prezzi dati”, si veda ad esempio Franco Donzelli, (2007). Ancora "Per sommi capi sembra lecito affermare che la teoria microeconomica, di cui l'equilibrio economico generale rappresenta il paradigma più diffuso e accettato internazionalmente, mira a spiegare, soprattutto in un ambito uniperiodale, come si determinano (idealmente) i prezzi di equilibrio di una miriade di beni.", si veda Piercarlo Nicola, (2007), p. 67.

Con riferimento all'equilibrio economico sembra giustificato prendere spunto dalla Fisica e tenere presente che tipo di misurazione abbia diretto effettivo interesse. Ad esempio nell'ambito della teoria cinetica dei gas non ha importanza la misura microscopica dell'energia cinetica della singola molecola, ma è essenziale la misura macroscopica della temperatura che esprime l'energia cinetica media delle molecole (si veda Fausto Borgonovi, "Procedimenti di misurazione, predicibilità e determinismo delle leggi della Fisica", sintesi inclusa in questa raccolta). In corrispondenza, sia pure con le dovute cautele e nella prospettiva di maggiori approfondimenti, nello studio dell'Equilibrio economico generale si può ritenere che, in senso astratto, non abbia diretto interesse la misura dell'Utilità percepita dal singolo consumatore nei confronti dell'acquisto di un particolare bene, dato che il sistema ne tiene conto implicitamente in media, attraverso il prezzo di acquisto, che, come la temperatura, diviene l'indicatore di stato globale, direttamente osservabile in modo preciso ed agevole.

\subsection{Valutazione dell'utilità soggettiva in decisioni sotto incertezza}

Si consideri un soggetto economico che deve scegliere fra le conseguenze incerte descritte con valori monetari, quindi di tipo cardinale, osservabili $x_{i} \in X$ dell'insieme finito $X=\left\{x_{1}, x_{2}, \ldots, x_{n}\right\}$. La teoria di von Neumann, Morgenstern e Savage tratta in modo assiomatico e concettualmente sottile, con riferimento alla nozione di probabilità, da intendere tipicamente di assegnazione soggettiva, appunto dell'utilità in uno schema che diremo di scelta con rischio. 
In termini estremamente semplificati se $x_{1}$ è la conseguenza meno preferita, $x_{n}$ quella massimamente preferita, si assume l'esistenza di una funzione di utilità $u(x)$ con i valori $u\left(x_{1}\right)=0$ e $u\left(x_{n}\right)=1$, in modo di stabilire una scala per l'utilità, e inoltre si definisce quale valore di utilità $u(x)$ di un valore intermedio, $x_{1}<x<x_{n}$, il valore medio dell'utilità risultante da un'ipotetica scommessa che porta al risultato $x_{1}$ con probabilità $(1-p)$ ed al risultato $x_{n}$ con probabilità $p$, cioè

$u(x)=(1-p) u\left(x_{1}\right)+p u\left(x_{n}\right)=p u\left(x_{n}\right)=p$

$0<p<1$ e tenuto conto delle posizioni $u\left(x_{1}\right)=0, u\left(x_{n}\right)=1$. L'espressione (11) comporta l'assegnazione ad $x$, dopo un confronto di merito e di coerenza soggettivi con l'utilità assegnata $\mathrm{a} x_{1}, x_{n}$, di un valore di utilità intermedio pari a $p, 0<p<1$.

$\mathrm{La}$ (11) consente di definire importanti nozioni. In primo luogo il valore cosiddetto di certezza equivalente. Si tratta di un valore $x^{*}$ tale che la sua acquisizione certa ha per il soggetto una utilità $u\left(x^{*}\right)$ eguale all'utilità della scommessa, cioè, per quanto detto, al valore medio di utilità proprio della scommessa. Se si assume che $u(x)$ sia una funzione monotona crescente, che riteniamo, per semplicità continua, si ha dunque, ad esempio, per $n=2$ :

$x^{*}=u^{-1}\left[(1-p) u\left(x_{1}\right)+p u\left(x_{2}\right)\right]=u^{-1}(p)$

dove $u^{-1}(\cdot)$ indica la funzione inversa di $u(x)$.

L'altra importante nozione è quella di avversione al rischio. Un soggetto è avverso al rischio se il valore certo, eguale alla media teorica o valore atteso della scommessa dicotomica vista sopra, indicato con $M(\bar{x})=\left[(1-p) x_{1}+p x_{2}\right]$, ha una utilità maggiore del valore di certezza equivalente, in simboli: $M(\bar{x})>x *=u^{-1}(p)$.

Ciò significa, in termini intuitivi, che il soggetto, dal punto di vista dell'utilità associata ai valori $x$, preferisce essere certo di ottenere il valore $M(\bar{x})$, pari alla media dei valori osservabili, piuttosto che ottenere con certezza il risultato $x^{*}$, che ha utilità pari a quella della media delle utilità dei valori, estremi convenzionali, osservabili ma aleatori.

La precedente definizione di avversione al rischio è facilmente estensibile al caso politomico in cui il valore atteso dei risultati della scommessa e delle rispettive utilità concerne gli $n$ valori $x_{i}, i=1,2, \ldots, n$, dell'insieme $X$. Generalizzando, infatti, la (11) si ha: 
$u\left(\sum_{i=1}^{n} p_{i} x_{i}\right)>\sum_{i=1}^{n} p_{i} u\left(x_{i}\right)$

dove $0<p_{i}<1, \Sigma_{i} p_{i}=1$, che stabilisce come l'utilità della media dei valori osservabili sia maggiore dell'utilità media degli stessi ed esprime quindi, in termini generali, l'avversione al rischio. Si dimostra che condizione necessaria e sufficiente per la validità della (12) è che la funzione $u(x)$ sia concava, si veda ad es. R.L. Keeney, H. Raiffa (1993), pp. 149 e seguenti.

La utilità media al secondo membro della (11) è assunta, in generale, quale valore dell'utilità di un valore intermedio $x, x_{1}<x<x_{2}$, in una scommessa dicotomica, con probabilità ( 1-p) e $p$ del verificarsi rispettivamente di $x_{1}$ o di $x_{2}$, ed è stata ampiamente utilizzata per stabilire sperimentalmente dei tipici andamenti della funzione di utilità, si veda ancora la fonte precedente, pp. 203 e seguenti. L'argomento, da ritenere rilevante nell'ambito delle decisioni economiche, con esplicita giustificazione razionale, è spesso menzionato, omettendo i particolari. Forse a scapito dell'uniformità di questa concisa presentazione di insieme, si aggiungono al riguardo alcuni dettagli.

Seguendo J.O. Berger, (1985), pp. 54 e seg., consideriamo - solo per fissare le idee - un "decisore" che guadagna 10.000 \$ all'anno ed ha un deposito bancario di $500 \$$. In vista di un investimento egli vuole stabilire, in base alle proprie valutazioni di convenienza, l'andamento della sua funzione di utilità nell'intervallo $[0,1000]$ utilizzando la tecnica di riferirsi a scommesse con due soli risultati, che stanno ad indicare degli eventi aleatori, diciamo di natura finanziaria, ritenuti inizialmente entrambi possibili con probabilità $p=1 / 2$. Si supponga che il decisore accetti che l'utilità del valore nullo, cioè, di $x_{1}=0$ sia $u(0)=0$, e quella del ricavo $x_{2}=1000$ sia $u(1000)=1$. Il punto importante successivo è quello di scegliere un terzo valore $x_{3}, 0<x_{3}<1000$, per il quale il decisore conviene di accettare il valore di utilità stabilito dalla relazione (11). Supponiamo che il decisore ritenga che l'utilità di $x_{3}=300$, inteso come valore certo, cioè $u(300)$, sia per lui la stessa di quella della scommessa - dove, pertanto, $x_{3}$ è il valore di certez$z a$ equivalente - e quindi, secondo la (11) egli possa ritenere

$u(300)=0.5 u(0)+0.5 u(1000)=0+0.5 \cdot 1=0.5$.

Il procedimento richiede di considerare due altri punti intermedi $x_{4}$ e $x_{5}$, rispettivamente negli intervalli $(0,300),(300,1000): 0<x_{4}<300,300$ 
$<x_{5}<1000$, con risultati collegati - nello stesso modo visto per $x_{3}-$ a due scommesse dicotomiche, entrambe con $p=0.5$.

Si considerino $x_{4}=100, x_{5}=500$. In base alla (11) si ottiene, per definizione, l'utilità

$u(100)=0.50+0.5 \cdot 0.5 .=0.25$;

però questo vale anche per un valore maggiore, ad esempio, $x_{4}=125$, cioè:

$u(125)=0.50+0.5 \cdot 0.5=0.25$

e il decisore, per la monotonicità della funzione $u(x)$, non può accettare la stessa utilità nei due casi. Supponiamo che egli ritenga più consono alla sua valutazione $u(125)=0.5 \cdot 0+0.5 \cdot 0.5=0.25$, mentre per la monotonicità anzidetta, dovrà essere $u(100)<u(125)$.

In modo simile si ottiene:

$u(500)=0.5 \cdot u(300)+0.5 \cdot u(1000)=0.5 \cdot 0.5+0.5 \cdot 1=0.75$

ma lo stesso valore si ottiene per un valore maggiore, diciamo 550.

Risulta, pertanto, coerente che il decisore ritenga che in effetti sia $u(500)<u(550)$ e più consono alla sua valutazione di utilità il ritenere $u(550)=0.75$. Il decisore può allora controllare se sussiste coerenza fra le valutazioni di utilità da lui effettuate, almeno nel senso che rispettano il carattere monotono crescente della funzione di utilità. A tale fine può essere utile considerare un ulteriore "punto intermedio" fra 100 e 300, diciamo 250.

Con riferimento alle consuete scommesse con $p=0.5$ si ottiene:

$u(250)=0.5 \cdot u(100)+0.5 \cdot u(300)<0.5 \cdot 0.25+0.5 \cdot 0.5=0.375$,

che è coerente con $u(300)=0.5$; si può inoltre controllare la coerenza dell'utilità, direttamente assunta come adeguata per $x=300$, osservando che quest'ultimo è un valore intermedio fra 125 e 550 . Applicando la (11) di nuovo si ha ancora:

$u(300)=0.5 \cdot u(125)+0.5 \cdot u(550)=0.5 \cdot 0.25+0.5 \cdot 0.75=0.5$.

La funzione di utilità sperimentale soggettiva dell'esempio in 
esame è, in definitiva, descritta dai valori sotto riportati, che delineano, se interpolati mediante una conveniente funzione continua, un andamento concavo.

Tabella 2. Esempio di funzione di utilità sperimentale.

\begin{tabular}{cccccc}
\hline$x$ & 0 & 125 & 300 & 550 & 1000 \\
\hline$u(x)$ & 0 & $1 / 4$ & $1 / 2$ & $3 / 4$ & 1 \\
\hline
\end{tabular}

Il pregio del metodo anzidetto, che è soggettivo ma sperimentale, e delle considerazioni teoriche che lo giustificano è di avere consentito di individuare dei tipici andamenti della funzione di utilità, quale quello "esponenziale negativo", per la cui verifica ne basta controllare l'adattamento alla situazione sperimentale, sulla base di una tecnica del tipo sopra delineato, per un limitato numero di valori $x$.

Di fronte a diverse situazioni di incertezza, cioè di possibili diverse distribuzioni di probabilità $p_{i}, i=1,2, \ldots, n$, dei risultati il decisore, con l'assunzione di essere caratterizzato da una funzione di utilità convoca, tenderà, se ne è in grado, a porsi nella situazione aleatoria che gli assicura il massimo valore medio di utilità, che può ritenersi comporti, in genere, anche dei valori medi elevati dei risultati monetari, in accordo alla (12). Per una revisione critica dell'argomento del rischio si suggerisce l'articolo di V.B. Frosini (1997).

\subsection{Misura psicometrica dell'Utilità}

Qualche cenno su questo metodo ampiamente utilizzato nel marketing.

Si considera un soggetto che attua una scelta di preferenza fra gli elementi di un insieme finito $X$ di alternative (ad esempio: diversi prodotti) nel quale ogni elemento è caratterizzato dalla modalità di un attributo, espressa su una scala almeno ordinale (ad esempio: soddisfacimento nei confronti di un prodotto). Si ammette che l'utilità sia descritta da una variabile aleatoria non direttamente osservabile o, come si suole dire, latente, diciamo $U_{x}, x \in X$. L'idea di base è che in una situazione concreta l'utilità che il soggetto associa allo stimolo proveniente dalla modalità $x$ di un generico elemento di scelta sia la determinazione di $U_{x}$ e che la sua scelta corrisponda a considerare il massimo di $U_{x}, x$ $\in X$. Da questa premessa si è sviluppata la ormai classica metodologia 
psicometrica di Thurstone dei confronti a coppie nella quale, nel caso più noto e importante, caso $V$, si assume che le variabili $U_{x}$, abbiano una distribuzione normale con la stessa varianza, mentre le medie possono essere diverse, e siano stocasticamente indipendenti. Lo sviluppo teorico porta, nel caso $V$, a potere esprimere e, quindi a stimare, il valore della variabile latente Utilità, attraverso la funzione inversa della funzione di ripartizione della variabile normale standardizzata $N(0,1)$, cioè su una scala ad intervalli.

Il metodo trova applicazioni, con modifiche e adattamenti, nelle ricerche di mercato al riguardo della cosiddetta conjoint analysis che comporta di chiedere a un soggetto il suo ordine di preferenza nei confronti di $n$ ipotetici prodotti fra essi diversi per le variazioni di $k$ fattori controllabili. Per ciascuno di questi ultimi (ad esempio, con riferimento ad un veicolo: prezzo di acquisto, consumo di carburante per $\mathrm{km}$., costo annuale dell'assicurazione) si riesce a stimare lo specifico contributo di utilità all'utilità complessiva dell'oggetto, si veda ad esempio $\mathrm{P}$. E. Green, D. S. Tull (1988), Cap. 15, ed anche A. Zanella, M. Cerri (2000).

\subsection{Possibile valutazione dell'Utilità secondo la metodologia tipica nelle scienze sociali}

Si è sviluppata, in tempi abbastanza recenti, una metodologia basata principalmente su aspetti di teoria statistica e probabilistica, rivolta alla "quantizzazione" di nozioni astratte o concetti. Precisamente la metodologia propone un procedimento di misurazione rivolto a stabilire una corrispondente rappresentazione numerica. Si veda al riguardo il trattato fondamentale di K. A. Bollen (1989), in particolare il Cap 6 . Alle pp. 180 e seg. dello stesso vengono riassunti gli aspetti principali del metodo che sotto si riassumono.

1) Il processo di misurazione inizia con la definizione verbale del concetto. Il concetto è un'idea che unifica delle manifestazioni fenomeniche (come attitudini, comportamenti, tratti caratteristici, ecc.): richiede una definizione teorica che illustri in modo semplice e preciso il significato del concetto (ad esempio: "terrorismo internazionale").

2) Segue l'individuazione delle dimensioni del concetto: queste sono aspetti distinti del concetto che, cioè, non è facile suddividere in ulteriori componenti (nel caso citato: sequestri di aerei, attentati dinamitardi, ecc.). Ad ogni dimensione si associa una variabile alea- 
toria non osservabile o latente.

3) Definizione operativa che consiste nell'associare ad ogni dimensione almeno due indicatori o variabili osservabili, atte a descrivere l'aspetto del concetto che definisce la dimensione .

4) Formulazione esplicita del modello statistico matematico che collega ogni dimensione con i propri indicatori osservabili e con errori aleatori non osservabili.

In corrispondenza la teoria probabilistica e statistica (ad esempio l'analisi dei fattori latenti) consente di pervenire, una volta completate in termini più tecnici le assunzioni, alla stima dei valori delle variabili latenti, si veda, ad es., la fonte precedente, p. 305.

Non mi è noto che sussistano degli studi sperimentali - come nel caso della Customer Satisfaction - nei quali venga esaminata e misuratala nozione di Utilità secondo la metodologia cui si è accennato sopra. Si noti come l'applicazione di tale metodologia richieda, in particolare, una definizione esplicita di Utilità - che non potrà ovviamente prescindere da contenuti etici - atta ad essere collegata con aspetti oggettivi direttamente osservabili.

\section{CONCLUSIONI}

Si è visto come per le grandezze economiche, considerate di tipo monetario, si tratta, in generale, di accertare, per un determinato periodo, l'ammontare totale o quello medio di una popolazione e si devono utilizzare per la loro stima i risultati di indagini campionarie.

L'errore medio di misura risulta, in corrispondenza, incrementato dalla variabilità dovuta al campionamento.

L'utilizzazione di schemi di campionamento efficienti assicura, però, che l'errore di misura di un totale o di una media aritmetica rimane in pratica trascurabile, rispetto al valore vero che può "localizzarsi" con sufficiente accuratezza. Si è visto come questo sia il caso, ad esempio, della spesa famigliare annuale media.

La situazione diviene diversa se si è interessati non ad accertare una situazione in un determinato periodo di tempo, ma si vogliono delle previsioni al riguardo di periodi futuri in base ad un modello economico ovvero econometrico. In questo caso l'errore di misura e di campionamento può risultare incrementato in modo non direttamente valutabile a causa della non esatta conoscenza del modello interpretativo o anche per l'impossibilità di realizzare gli interventi correttivi sul 
sistema economico suggeriti dal modello. Un caso tipico può ravvisarsi nelle previsioni sull'indice dell'inflazione.

Si è quindi esaminata la misura dell'utilità in condizioni di equilibrio di una economia di mercato. Questa può collegarsi alle scelte dei soggetti economici, cioè, in definitiva, alle scelte di individui che operano in accordo ad un'entità difficilmente definibile in termini generali e pertanto difficilmente misurabile, appunto indicata come Utilità. Può avere scarso interesse generale la misura dell'utilità per un singolo consumatore e si può invece pensare che lo abbia qualche indicatore globale, che può in qualche modo ritenersi in relazione - ciò che è argomento da approfondire - ai prezzi di mercato.

Di fronte ad una pluralità di alternative soggette ad incertezza, che è la situazione tipica del "decisore" in ambito finanziario e di impresa, la teoria di von Neumann-Morgenstern-Savage può essere utile per orientare verso scelte che massimizzano l'utilità media rispetto alle distribuzioni di probabilità ipotizzate per le possibili conseguenze.

Si è anche visto che, nella ricerca di mercato, la formulazione della nozione di utilità diviene più concreta e le tecniche psicometriche consentono, in corrispondenza, di individuare il prodotto più utile.

Si è infine ricordato come è disponibile una metodologia statistico-probabilistica per la quantizzazione dei concetti della quale si deve ancora approfondire l'impiego per la valutazione dell'utilità.

In definitiva, di fronte al quesito posto dal Convegno, l'analisi statistica porta a concludere che il completamento della teoria economica e dello studio di adeguati modelli econometrici, integrati da conseguenti possibili interventi di politica economica - ferma restando la libertà ed autonomia presente negli operatori economici che rimangono una componente fondamentale del sistema economico - potrà ragionevolmente consentire qualche passo ulteriore per un avvicinamento ad una predicibilità paragonabile a quella possibile nell'ambito delle scienze naturali. 


\section{BIBLIOGRAFIA}

Banca d'Italia: Relazione Annuale, Bollettino Statistico.

ISTAT: Annuario, Bollettino mensile di Statistica.

Berger J. O., (1985), Statistical Decision Theory and Bayesian Analysis, Springer Verlag, Berlin, Cap.2.

Biemer P.P., Trewin D. (1997), "A Review of Measurement Error Effects on the Analysis of Survey Data "in A.A.V.V. Survey Measurement and Process Quality, Wiley, New York, pp. 603-632.

Blum J., (1983), "Sampling Errors (Computation of)", in S. Kotz, N. Johnson, Eds. Encyclopedia of Statistical Sciences, Vol II, pp.240-244.

Bollen K.A., (1989) Structural Equations with Latent Variables, Wiley, New York.

Borgonovi F., "Procedimenti di misurazione, predicibilità e determinismo delle leggi della Fisica", sintesi inclusa in questa raccolta.

Donzelli F., (2007), "Equilibrio, Disequilibrio e Tempo in Walras", A.A.V.V., Incontri di studio, Economia Matematica e Econometria, Istituto Lombardo - Accademia di Scienze e Lettere, Milano, p.31.

Frosini V.B., (1997), "The Evaluation of Risk Attitudes: a New Proposal", Statistica applicata, Vol. 9.11.4, pp. 435-458.

Green P.E., Tull D. S., (1988), Research for Marketing Decisions, $5^{\circ}$ Ed., Prentice Hall, New York, Cap. 15.

Keeney R.L., Raiffa H., (1993), Decisions with Multiple Objectives: Preferences and Value Tradeoffs, Cambridge University Press.

Krantz D. H., Luce R., Suppes P.,Tversky A., (1971), Foundations of Measurement, Academic Press, New York, Cap.1.

Marigliani M., (2004), L'indagine trimestrale ISTAT sui consumi delle famiglie italiane. Natura ed utilizzi nell'ambito della misurazione della povertà e nella costruzione di aggregati di contabilità nazionale. Agenzia delle entrate, Documenti di Lavoro 2004/1 disponibile on-line).

Nicola P., (2007), "Fondamenti di Macroeconomia dinamica", A.A.V.V., Incontri di studio, Economia Matematica e Econometria, Istituto Lombardo - Accademia di Scienze e Lettere, Milano, p.67.

Rapacciuolo C:, (2003), Un semplice modello univariato per la previsione a breve termine dell'inflazione italiana, Centro Studi Confindustria, n.36, pure disponibile su web.

Roffia V., ed A. Zaghini A., (2008), Excess Money Growth and Inflation Dynamics, n.657, disponibile su web.

Scannapieco M., Zardetto D., e G. Barcarol G., (2007), La calibrazione dei dati con R: una Sperimentazione sull'indagine Forze di lavoro e un confronto con GENESEES/SAS, Contributi ISTAT, n. 4/2007.

Zanella A., Cerri M., (2000), "La misura di Customer Satisfaction: qualche riflessione sulla scelta delle scale di punteggio" in A.A.V.V.,Vita e Pensiero, Milano, pp. 217-231. 